\title{
Erratum: Generation and monitoring of directed neutrino beams using electron-capture $\beta$-decay sources [Phys. Rev. C 86, 034615 (2012)]
}

\author{
C. DeAngelis, L. M. Folan, and V. I. Tsifrinovich
}

(Received 17 September 2018; revised manuscript received 5 October 2018; published 13 March 2019)

DOI: 10.1103/PhysRevC.99.039902

In the article, the authors pointed out an opportunity to generate directed monoenergetic neutrino beams using electron-capture $\beta$ decay if atomic nuclei, polarized by a strong hyperfine field, decay with decreasing nuclear spin by one unit. It was suggested to observe this effect by attaching a radioactive sample to a cantilever tip and measuring the recoil force with atomic force microscopy. The recoil force was estimated assuming that the neutrino can propagate only along the axis of polarization.

In this Erratum, we would like to clarify that the generated neutrino beam does not have a unique direction of propagation but rather has the preferred direction of propagation: The probability of neutrino emission is minimum in the direction of nuclear polarization and maximum in the opposite direction. Also, we present a more accurate expression for the recoil force.

We assume that nuclei are completely or partially polarized in the positive $z$ direction. Then the net recoil force $F$ on the radioactive sample is associated with the rate $\dot{P}_{z}$ of the generation of the neutrino momentum,

$$
F=-\int_{0}^{2 \pi} d \phi \int_{0}^{\pi} \dot{P}_{z}(\theta) W(\theta) \sin (\theta) d \theta .
$$

In this formula, $\theta$ is the polar angle of the recoil force produced by an individual neutrino, $W(\theta)$ is the probability density, and the integral is taken over all emission angles. The assumed strong magnetic-field $B$ breaks the symmetry, but the emissions are still symmetric in the azimuthal angle $\phi$. Next, we have

$$
\dot{P}_{z}(\theta)=-\dot{P} \cos \theta, \quad \dot{P}=\dot{E} / c=\alpha E_{v} / c .
$$

Here $\dot{E}$ is the power of the neutrino radiation, $\alpha$ is the decay rate (activity) of a radioactive sample, and $E_{v}$ is the energy of an individual neutrino. Using an expression for angular distribution of the recoil force derived by Treiman [1] and the values of the coupling constants from Ref. [2], we obtain the approximate expression for the probability density $W(\theta)$,

$$
W(\theta)=\frac{1}{4 \pi}\left\{1+B_{I}(q) \cos \theta\right\} .
$$

Here $B_{I}(q)$ is the Brillouin function, $q=\mu B / k_{B} T$, $I$ and $\mu$ are the spin and magnetic moment of the parent nuclei, $B$ is the sum of the hyperfine and external magnetic fields, and $T$ is the temperature of the spin system. Finally, computing the integral in Eq. (1) we find the sought-for expression for the net recoil force,

$$
F=\frac{\ln (2)}{3 T_{1 / 2}} \frac{E_{v}}{c} N B_{I}(q) .
$$

where $T_{1 / 2}$ is the half-life and $N$ is the number of radioactive atoms.

The recoil force computed here is three times smaller than that obtained in the original article in the low-temperature limit. There is also a typographic error in the expression for $\dot{E}$ in the paragraph preceding Eq. (1) of the original article. It should read $\dot{E}=\alpha E_{v}=\ln (2) N E_{\nu} / T_{1 / 2}$.

The authors gratefully acknowledge the assistance of R. Basak.

[1] S. B. Treiman, Phys. Rev. 110, 448 (1958).

[2] H. Paul, Nucl. Phys. A 154, 160 (1970). 